\title{
DECOMPENSATION OF CHRONIC OPEN ANGLE GLAUCOMA FOLLOWING A SPINAL STEROID INJECTION: A CASE REPORT
}

\author{
Julinda Jaho $^{1 *}$, Suzana Nuellari², Artan Bano ${ }^{3}$ \\ I* Ophthalmology Department, University Hospital of "Mother Theresa" \\ and German Eye Clinic, Tirana, Albania; \\ ${ }^{2}$ Service of Ophthalmology, Policlinic of Specialties Nr. 3,Tirana, Albania; \\ ${ }^{3}$ Orthopaedic Department, University Trauma Hospital, \\ Tirana, Albania;
}

*Corresponding Author Julinda Jaho, e-mail: julindajaho@gmail.com;

Received October 2021; Accepted November 2021; Published January 2022;

DOI: https://doi.org/10.31407/ijees12.103

\begin{abstract}
Corticosteroids are widely used in many different medical conditions. Increased intraocular pressure (IOP) is one of their side effects. We are presenting the case of a patient, female, 58 years old, who presented to our clinic with blurred vision in the right eye. Intraocular pressure was $33 \mathrm{mmHg}$ in the right eye and $12 \mathrm{mmHg}$ in the left eye. She had a history of controlled primary open angle glaucoma under conservative treatment in the right eye. Both eyes had undergone trabeculectomy in the past. The left eye had an advanced primary open angle glaucoma which had been controlled after the Trabeculectomy without any topical medication. She reported a history of a spinal steroid injection 3 weeks ago. Despite the immediate and prolonged use of antiglaucomatous medications, the intraocular pressure did not come back to normal levels, leading to further damage of the optic nerve in this eye. A right eye glaucoma surgery was performed, which stabilized the IOP. The IOP of the left eye remained unaffected. Considering this as a case of steroid induced decompensation of glaucoma, we think that clinicians should inform patients about the possibility of visual complications associated with steroid injections. This would be of great importance especially for patients already having glaucoma or ocular hypertension and for patients who have a family history of glaucoma. Ophthalmologists should also alert glaucoma patients for the possibility of decompensation of the disease from such injections. Nevertheless, we believe that further studies are needed to quantify the amount of IOP elevations following different types of steroid injections.
\end{abstract}

Keywords: glaucoma, intraocular pressure, corticosteroids, spinal injection. 